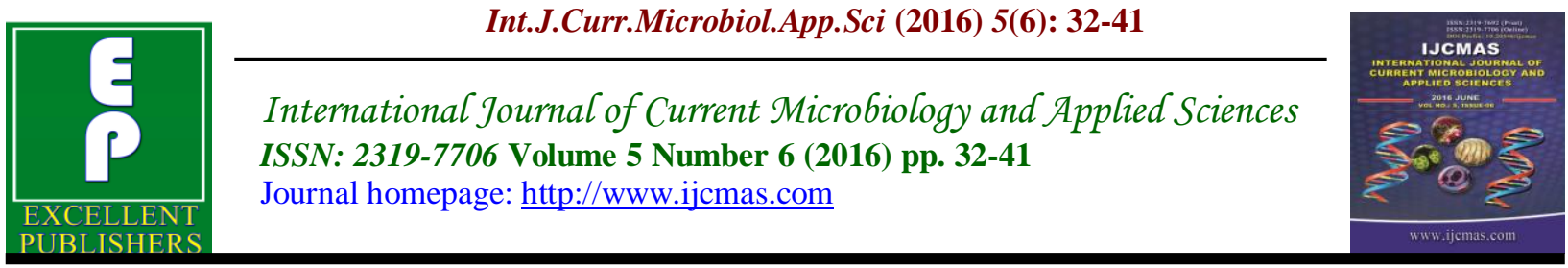

Original Research Article

http://dx.doi.org/10.20546/ijcmas.2016.506.004

\title{
Isolation and Characterization of Bacteria and Yeast from Kombucha Tea
}

\author{
Tehmeena Ashraf Mukadam*, Kapil Punjabi, Sunita D Deshpande, \\ Shashikant Prabhakar Vaidya and Abhay Shadashiv Chowdhary
}
Clinical Pathology Department, Haffkine Institute for Training, Research and Testing, Acharya, Donde Marg, Parel, Mumbai, India
*Corresponding author

\section{A B S T R A C T}

Keywords

Acetobacter, Kombucha, lichen, yeast, Acetic acid bacteria.

\section{Article Info}

Accepted:

07 May 2016

Available Online:

10 June 2016
Kombucha is a sugared tea fermented with flat, pancake like culture of yeast and acetic acid bacteria (AAB), which is consumed worldwide for its refreshing and beneficial effects. In this study Kombucha culture from a fermented tea was screened for the presence of $\mathrm{AAB}$ and yeast using selective media. Biochemical analysis for identification and confirmation of the genus of the bacteria and yeast were performed, followed by molecular characterization using partial sequencing targeting conserved sequences of both organisms. Biochemical analysis confirmed presence of organisms belonging to genus Acetobacter. Yeast was unidentified by biochemical analysis. Molecular characterization of the isolates identified acetic acid bacteria as Komagataeibacter saccharivorans and yeast as Zygosaccharomysis bailli.

\section{Introduction}

Kombucha a fermented tea beverage known worldwide by many, originated in China which later spread to Russia and rest of the world. Microbiologist earlier had classified Kombucha as lichen based on the fact that Kombucha is symbiotic association of fungi and AAB. However, typical lichen is a symbiosis of algae and fungi and required light to carry out photosynthesis, unlike Kombucha that grow in dark (Grow youthful health at any age, 2014; Velićanski et al., 2014).

Kombucha culture appears as a white rubbery pancake and appearance changes during fermentation period. Initially it is creamy white colour but when brewed in black tea the culture darken due to presence of tannins in tea.

Mushroom culture is steeped in sugared tea for fermentation. During this process original mushroom floats in the tea and baby mushroom is produced on the surface of the original culture. This new mushroom can now be used as culture for further process of fermentation (Jayabalan et al., 2014).

In various studies Kombucha is found to be a symbiotic association of bacteria such 
Acetobacter xylinoides, A. pasteurianus, A.xylinum, A. aceti, and Bacterium gluconicum and yeast like Schizosaccharomyces pombe, Saccharomycodes ludwigii, Saccharomyces cerevisiae, Zygosccharomyces bailli, Brettanomyces bruttanomyces, B. bruxellensis, B.lambicus, B.custersii and pichia species (Chen \& Liu, 2000; Fu et al., 2014). The exact microbial composition also depends on the source of the innocula of the tea fermentation. Bacteria and fungus in Kombucha present powerful symbiosis which are able to inhibit the growth of potential contaminating bacteria (Dufresne \& Farnworth, 2000).

Due to the variability of Kombucha, the bacteria and yeast present in given Kombucha from a certain source can vary at large. Due to this variability the fermentation of tea and its properties tend to differ. Thus it is important to identify the organisms present in the Kombucha used from a certain source for tea fermentation.

In the present study, the objective thus remains to isolate and identify the organisms present in the Kombucha that has been procured from a source and is being used for tea fermentation.

\section{Material and Methods}

\section{Tea Preparation}

Branded tea was used for the preparation of Kombucha tea. $2 \%$ tea was added to boiling water and allowed to infuse for 5 minute. The infusion was filtered through a sterile sieve. $10 \%$ sucrose was dissolve in hot tea and preparation was left to cool. $10 \%$ previously fermented liquid tea broth was aseptically added in to the fresh tea. The tea was then poured in sterile glass bottles to which 3\% freshly grown tea fungus that had been previously cultured in the same medium for 28 days was added. The bottles were covered with sterile muslin cloth and fastened tight.The fermentation was carried out at room temperature (RT) in static condition and in dark for 28 days (Jayabalan et al., 2008).

\section{Isolation of Bacteria and Yeast}

Enrichment and Isolation techniques were employed for determining the bacteria and yeast present in Kombucha that was used for fermentation of tea. The enrichment media used was Glucose yeast extract broth (GY). Inoculated broth was incubated at room temperature for 48 hours. Isolation was carried out on two different selective media for isolation of Acetic acid bacteria, Glucose-ethanol medium (GEM) and Glucose yeast extract calcium carbonate medium (GYC). Isolation of yeast was carried out using Sabouraud Dextrose agar (Hi-Media Laboratories Pvt. Ltd., India). All Plates were incubated for 7 days at room temperature (RT) (Hanmoungjai et al., 2007; Soheir \& El-Salam, 2012)

\section{Biochemical Characterization of Acetic Acid Bacteria}

The morphology and Gram nature of acetic acid bacteria isolated on the selective media was determined. Its biochemical characterization involved catalase, oxidase, over oxidation of ethanol by use of Carr medium, oxidation of acetate and oxidation of lactate (Amoa-Awua et al., 2007; Asai et al., 1964; Kadere et al., 2008; Maal \& Shafiee, 2010)

\section{Biochemical Characterization of Yeast}

The morphology and microscopic analysis of yeast isolated on Sabouraud Dextrose agar (HiMedia Laboratories Pvt. Ltd., Mumbai) was determined. Biochemical characterization was carried out involving 
Isolation on HiCrome Candida Differential Agar (HiMedia Laboratories Pvt. Ltd., Mumbai), Germ tube test and urease production test (Marinho et al., 2010; Salviya et al., 2015). Identification of yeast other than Candida was performed by ethanol tolerance test (Guimarães et al., 2006). Acetic acid tolerance test was also performed using acidified media (Kurtzman et al., 2001).

\section{Molecular Characterization}

Identification of organisms by phenotypic and biochemical analysis is intricate and time consuming, thereby making molecular characterization an easy, precise and a reliable technique. The molecular method employed was partial sequencing using 16S rRNA gene and 18S rRNA gene as targets for the identification of bacteria an yeast respectively and the sequence was analysed using BLAST (Nithya \& Bhaskar, 2013; Soheir \& El-Salam, 2012). The amplification and sequencing of the bacteria and yeast was outsourced to GeneOmbio Technology Pvt. Ltd.

\section{Results and Discussion}

\section{Fermentation of Tea}

Fermentation of tea gets initiated after adding inoculums of Kombucha $(10 \% \mathrm{v} / \mathrm{v})$ along with piece of pan cake $(3 \% \mathrm{w} / \mathrm{v})$ in prepared black tea. As growth proceeds the new growing culture starts to form its own pancake layer. During this period, visible changes in the tea observed are typical fermentation odour and formation of gas bubbles as result of $\mathrm{CO}_{2}$ generation. Over a period of 28 days the culture darkens due to presence of tannins in black tea and Kombucha cake grows up to cover the entire circumference of the glass bottle.

\section{Isolation of Bacteria \& Yeast}

The enriched broth after incubation of $48 \mathrm{hrs}$ showed turbidity indicating presence of organisms in the sample. Isolated colonies with zone of clearance around them after incubation at room temperature on GYC and GEM selective media confirmed the growth of acetic acid bacteria (Fig. 1). Similarly white colonies with yeast like morphology on Sabouraud Dextrose agar at room temperature were confirmed as Yeast.

\section{Biochemical Characterization of Acetic Acid Bacteria}

The isolate was Gram negative short rod, catalase positive and oxidase negative. Complete oxidation of acetate and lactate was observed with no brown pigmentation on GYC medium. An initial colour change from green to yellow and reversion to green colour on further incubation was observed on the Carr medium due to the growth of the isolate (Fig. 2) (Table 1).

\section{Biochemical Characterization of Yeast}

Isolate obtained from Sabouraud Dextrose agar was Gram positive oval budding yeast. It showed pink coloured colonies on HiCrome Candida Differential Agar while urease production and germ tube test were negative. The organism was found to tolerate ethanol and acetic acid in respective media (Table 2).

\section{Molecular Characterization}

Partial nucleotide sequences of Acetic acid bacteria and yeast have been represented in Fig. 3 and Fig. 4. Sequencing analysis obtained was subjected to BLAST. BLAST results illustrated that the test bacterium was similar to Komagataeibacter saccharivorans strain with $100 \%$ identity, query cover and 
0.0 E value. The yeast exhibited $99 \%$ identity with $0.0 \mathrm{E}$ value to Zygosaccharomyces bailii.

Kombucha is refreshing beverage well known for its health beneficial effects. Kombucha is a fermented tea which is obtained by inoculating sweetened tea with acetic acid bacteria and yeast (Blanc, 1996; Jayabalan et al., 2010). After 10 to 14 days of inoculation a new tea fungus develops on the surface of tea. Kombucha tea consists of two portion cellulosic pellicle layer which floats at the top of broth and sour liquid broth. As fermentation proceeds characteristic odour of fermentation and gas bubbles formed due to carbolic acid produced are observed (Chen \& Liu, 2000; Jayabalan et al., 2014). These observations are typical of growth of Kombucha culture and indicative of fermentation of tea (Jayabalan et al., 2014).In present study, observation noted regarding signs of fermentation cycle is similar to study reported by Jayabalan et al., thus it can be concluded that fermentation cycle carried out in present study was successful.

Isolated colonies on GYC and Sabouraud Dextrose agar was identified as $\mathrm{AAB}$ and yeast. Soheir et al., Stated that Acetic acid bacteria shows clear halo after acidification on GYC medium (Soheir \& El-Salam, 2012). Isolated strain exhibited similar result, thus confirmed as $\mathrm{AAB}$.

According to Kadare et al isolate classified under Acetobacter genera are catalase positive, oxidase negative, oxidize lactate and acetate to $\mathrm{CO} 2$ and $\mathrm{H} 2 \mathrm{O}$. Acetobacter strains are able to overoxidise ethanol to acetic acid and finally to $\mathrm{CO} 2$ and $\mathrm{H} 2 \mathrm{O}$.As tricarboxylic acid cycle is functional in Acetobacter strain, genera is able to overoxidise organic acids. Ability to oxidise acetic acid to $\mathrm{CO} 2$ is a major distinguishing feature between genera Acetobacter and Gluconobacter (Kadere et al., 2008; Ukwo \& Ezeama, 2011). In the present study similar results were observed, therefore isolated strain in present study was confirmed Acetobacter.

As literature reports, in Kombucha various yeast cultures are present belonging to genus Candida and/or Saccharomyces (Jayabalan et al., 2014). Thus isolated strain was subjected for germ tube test which was found to be negative. This indicates presence of non Candida albicans (NCA). To distinguish between Candida Spps HiChrome Candida Differential Agar was used (Marinho et al., 2010). Candida Differential Agar which, as per manufacturer's literature indicates presence of Candida krusei. For confirmation of Candida krusei, urease test was performed which was negative signifying possibility of presence of other genus of yeast in the culture (Deorukhkar \& Saini, 2014). For further identification Acid and Ethanol tolerance test were performed. Isolated yeast was found to have Ethanol and Acid tolerance ability. Thus, indicating of presence of genus Saccharomyces.

Identification based on genotypic method is considered to be more accurate as compared to phenotypic assays. It is recognized that comparison of a stable part of genome can aid in determination of phylogenetic relationship and identification of organisms. Comparison of 16S rRNA and 18S rRNA is one of the preferred techniques for identification of bacteria and fungi respectively, especially in poorly described, rarely isolated and phenotypically inconclusive strains (Clarridge, 2004). 
Table.1 Biochemical Characterization of AAB isolate from Kombucha

\begin{tabular}{|l|l|}
\hline Biochemical test & Result \\
\hline Gram nature & Game negative short rods \\
\hline Carr medium & $\begin{array}{l}\text { Isolates producing acid - characteristic change in colour } \\
\text { of media observed (From Green to Yellow then Green } \\
\text { again) }\end{array}$ \\
\hline Catalase & + \\
\hline Oxidase & - \\
\hline Oxidation of acetate & + \\
\hline Oxidation of lactate & + \\
\hline Brown pigmentation on GYC & - \\
\hline Ker
\end{tabular}

Key: $+=$ positive, - = negative

Table.2 Biochemical Characterization of Yeast isolated from Kombucha

\begin{tabular}{|l|l|}
\hline Biochemical test & Result \\
\hline Gram nature & Gram positive oval budding yeast \\
\hline Germ tube & - \\
\hline Growth on Hichrome Agar & Pink colour colony \\
\hline Urease test & - \\
\hline Ethanol tolerance $\mathbf{1 0 \% , 1 3 \% ~ \& ~ 1 5 \%}$ & + (for all concentrations) \\
\hline Tolerance to acetic acid test & + \\
\hline
\end{tabular}

Key: $+=$ positive, $-=$ negative

Fig.1 Growth on GYC media




Fig.2 Change in Colour of Carr Media due to Growth of Acetobacter

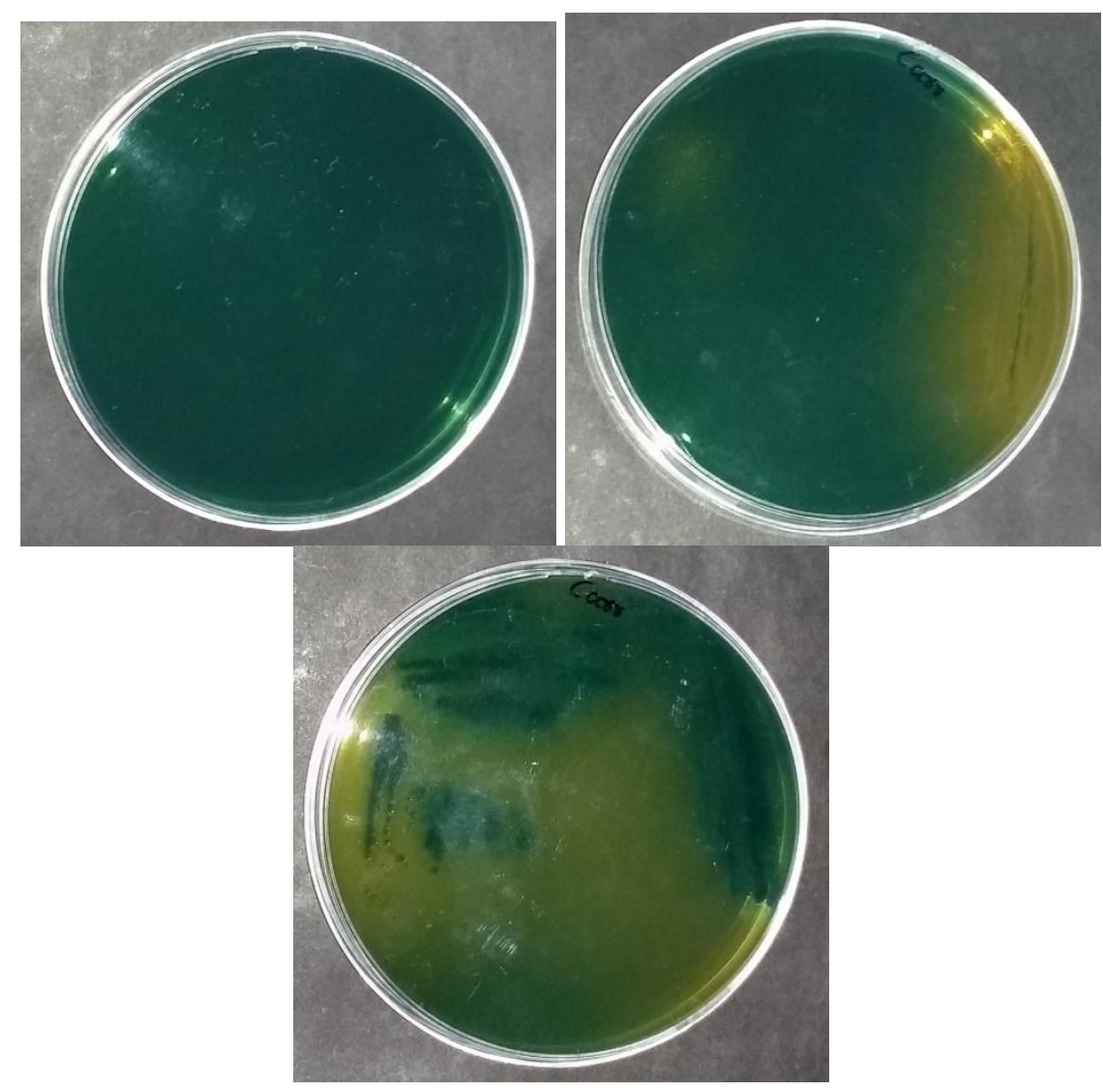

Fig.3 Nucleotide Sequence of AAB

$>2221(16 \mathrm{~S} 2)$

GCCAGGTTGCCGCCTTCGCCACCGGTGTTCTTCCCAATATCTACGAATTTCACCT CTACACTGGGAATTCCACAACCCTCTCTCACACTCTAGTCTCAACGTATCAAATG CAGCCCCCAGGTTAAGCCCAGGAATTTCACATCTGACTGTTAAAACCGCCTACGC GCCCTTTACGCCCAGTCATTCCGAGCAACGCTTGCCCCCTTCGTATTACCGCGGC TGCTGGCACGAAGTTAGCCGGGGCTTCTTCTGCGGGTACCGTCATCATCGTCCCC GCTGAAAGTGCTTTACAATCCGAAAACCTTCTTCACACACGCGGCATTGCTGGAT CAGGCTTGCGCCCATTGTCCAATATTCCCCACTGCTGCCTCCCGTAGGAGTCTGG GCCGTGTCTCAGTCCCAGTGTGGCTGATCATCCTCTCAGACCAGCTATCGATCAT CGCCTTGGTAGGCCTTTACCCCACCAACTAGCTAATCGAACGCAGGTTCCTCCAC AGGCGACTTGCGCCTTTGACCCTCAGGTGTCATGCGGTATTAGCTTCAGTTTCCC AAAGTTATCCCCCACCCATGGACAGATCCCTACGCGTTACTCACCCGTCCGCCAC TAACCCCGAAAGGTTCGTGCGACTTGCATGTGTTAAGCATGCCGCCAGCGTTCGC TCTGAGCCAGGA 
Fig.4 Nucleotide Sequence of Yeast

$>2222($ ITS4)

TCCCTAAAGTCCCCTCTGTTCTTTCGGAGTCGGTAAAACCTAATACGACATTTGG TTAGGAAAGAGGAGAGCAAGACGTTTCCGCCTAAAACTCACCCACACGCGTTCC CCAAAAGGCTTGCAATTTCAAGTTAACCCAAATGAAAAACAGAGTATCACTCAC TACCAAACACGAATGTTTGAGAAGGAAATGACGCTCAAACAGGCATGCCCCCTG GAATACCAAGGGGCGCAATGTGCGTTCAAAGATTCGATGATTCACGGAATTCTG CAATTCACATTACGTATCGCATTTCGCTGCGTTCTTCATCGATGCGAGAACCAAG AGATCCGTTGTTGAAAGTTTTGAATATTTTGTTTTTTAGTATTCGTTTTTGACTGT AATATTGAAAAAAAAAAAAAAAAATTTGTTGGGTTTTTACCTTTGGGGAGGGG

The 16S rRNA and 18S rRNA sequences obtained, were compared with that of other bacterial and yeast sequences in the GenBank using NCBI BLAST tool (http://www.ncbi.nlm.nih.gov/BLAST/Blast. cgi) to check its relationship and similarity. Based on sequence similarity, the AAB was identified as Komagataeibacter saccharivorans with $100 \%$ similarity. While the yeast was identified as Zygosaccharomyces bailii with $99 \%$ similarity.

Most commonly reported acetic acid bacteria include Acetoacter aceti, Acetoacter pasteurianus, Acetoacter xylinoides and Gluconoacetobacter xylinus. While the commonly identified yeast include Brettanomyces bruxelensis, Brettanomyces intermedius, Candida famata, Pichia membranofaciens, Saccharomyces cervisiae, Sachharomycodes ludwigii, Schizosacharomyces pombe, Torulaspora delbrueckii, Zygosaccharomyces bailli and Zygosaccharomyces Rouxii (Genette Belloso-Morales, 2003; Markov et al., 2006; Sievers et al., 1996).

The bacterial genera Acetobacter and Gluconobacter are predominant prokaryotes in Kombucha culture (Jayabalan et al., 2014). Mayser et al reported Acetobacter xylinum as a primary bacterium and yeast composition is highly variable but
Saccharomyces,

Brettanomyces, Zygosaccharomyces occur most frequently observed to be part of Kombucha culture (Mayser et al., 1995). Komagataeibacter rhacticus AF1(formerly known as Gluconacetobacter rhaeticus) was isolated from tea (Santos et al., 2014). Komagataeibacter is decendent of Acetobacter (Lisdiyanti et al., 2006; Yamada et al., 2012). In the present study, Komagataeibacter saccharivorans was isolated which belongs to same genera.

In this study twin cultures of the Kombucha were successfully identified as Komagaeibacter saccharivorans and yeast Zygosacchromyces bailli.

In conclusion, the microbial composition of Kombucha differs depending upon the climatic, geographical area or source. Also use of different starting material (sugar and tea) and a different starter culture will lead to a variation in fermentation process and thereby formations of variable end products or metabolites which might show a difference in activity. Hence, it becomes necessary to identify the organism present in Kombucha. In this study twin cultures of the Kombucha were successfully identified in the study as Komagaeibacter saccharivorans and yeast Zygosacchromyces bailli. In future chemical characterization and assessment of 
biological activity of fermented tea can be executed to understand the fermentation cycle and its beneficial effects on human health.

\section{References}

Amoa-Awua, W.K., Sampson, E., TanoDebrah, K. 2007. Growth of yeasts, lactic and acetic acid bacteria inpalm wine during tapping and fermentation from felled oil palm (Elaeis guineensis) in Ghana. J. Appl.Microbiol., 102(2): 599-606.

Asai, T., Iizuka, H., Komagata, K. 1964. The Flagellation and Taxonomy of genera Gluconobacter and Acetobacter with reference to the existence of Intermediate Strains. $J$. Gen. Appl. Microbiol., 10(2).

Blanc, P.J. 1996. Characterization of the tea fungus metabolites. Biotechnol. Lett., 18(2): 139-142.

Chen, C., Liu, B. 2000. Changes in major components of tea fungus metabolites during prolonged fermentation. J. Appl. Microbiol., 89(5): 834-839.

Clarridge, J.E. 2004. Impact of $16 \mathrm{~S}$ rRNA Gene Sequence Analysis for Identification of Bacteria on Clinical Microbiology and Infectious Diseases. Clin. Microbiol. Reviews, 17(4): 840-862.

Deorukhkar, S.C., Saini, S. 2014. Laboratory approach for diagnosis of candidiasis through ages. Int. J. Curr. Microbiol. App. Sci., 3(1): 206-218.

Dufresne, C., Farnworth, E. 2000. Tea, Kombucha, and health: a review. Food Res. Int,. 33(6): 409-421.

Fu, C., Yan, F., Cao, Z., Xie, F., Lin, J. 2014. Antioxidant activities of kombucha prepared from three different substrates and changes in content of probiotics during storage.

Food Sci. Technol., (Campinas). 34(1).

Genette Belloso-Morales, H.H.S. 2003. Manufacture of a beverage from cheese whey using a "tea fungus" Fermentation.

Revista Latinoamericana de Microbiología. 45(1-2).

Grow youthful health at any age. 2014. Kombucha, recipe, history, benefits, starter. How to make kombucha Retrieved 28 March 2014, from http://www.growyouthful.com/recipi es/kombucha.php

Guimarães, T.M., Moriel, D.G., Machado, I.P., Picheth, C.M.T.F., Bonfim, T.M.B. 2006. Isolation and characterization of Saccharomyces cerevisiae strains of winery interest. Revista Brasileira de Ciências Farmacêuticas. Brazilian J. Pharma. Sci., 42.

Hanmoungjai, W., Chukeatirote, E., Pathom-aree, W., Yamada, Y., Lumyoung, S. 2007. Identification of Acidotolerant Acetic Acid Bacteria Isolated from Thailand Sources. Res. J. Microbiol., 2: 194-197.

Jayabalan, R., Malba`sa, R.V., Lon`car, E.S., Vitas, J.S., Sathishkumar, M. 2014. A Review on Kombucha Tea-Microbiology,Composition, Fermentation, Beneficial Effects,Toxicity, and Tea Fungus. Comprehensive Reviews in Food Science and Food Safety, Vol.13.

Jayabalan, R., Malini, K., Sathishkumar, M., Swaminathan, K., Yun, S.E. 2010. Biochemical characteristics of tea fungus produced during kombucha fermentation. Food Sci. Biotechnol., 19(3): 843-847.

Jayabalan, R., Marimuthu, S., Thangaraj, P., Sathishkumar, M., Binupriya, A.R., Swaminathan, K., Yun, S.E. 2008. 
Preservation of Kombucha Tea Effect of Temperature on Tea Components and Free Radical Scavenging Properties. J. Agric. Food Chem., 56: 9064-9071.

Kadere, T.T., Miyamoto, T., Oniang`o, R.K., Kutima, P.M., Njoroge, S.M. 2008. Isolation and identification of the genera Acetobacter and Gluconobacter in coconut toddy (mnazi). African J. Biotechnol., 7(1684-5315 ): 2963-2971.

Kurtzman, C.P., Robnett, C.J., BasehoarPowers, $\quad$ E. 2001. Zygosaccharomyces kombuchaensis, a new ascosporogenous yeast from 'Kombucha tea'. FEMS Yeast Res., 1: 133-138.

Lisdiyanti, P., Navarro, R.R., Uchimura, T., Komagata, K. 2006. Reclassification of Gluconacetobacter hansenii strains and proposals of Gluconacetobacter saccharivorans sp. nov. and Gluconacetobacter nataicola sp. nov. Int. J. Systematic and Evol. Microbiol., 56(9): 21012111.

Maal, K.B., Shafiee, R. 2010. Isolation and Characterization of an Acetobacter Strain from Iranian White-Red Cherry as a Potential Strain for Cherry Vinegar Production in Microbial Biotechnology. Asian J. Biotechnol., 2: 53-59.

Marinho, S.A., Teixeira, A.B., Santos, O.S., Cazanova, R.F., Carlos, Ferreira, A.S., Oliveira, S.D. 2010. Identification of Candida spp. by phenotypic tests and PCR. Brazilian J. Microbiol., 41: 286-294.

Markov, S., Cvetković, D., Bukvić, B. 2006. Use of Tea fungus isolate as starter culture for isolate as starter culture fo obtaining of Kombucha. Annals of the faculty of engineering Hunedoara.
Mayser, P., Fromme, S., Leitzmann, G., Gründer, K. 1995. The yeast spectrum of the 'tea fungus Kombucha'. Mycoses. 38: 289-295.

Nithya, V., Bhaskar, A. 2013. 16S and 18S rRNA gene Sequencing Of Atypical Clinical Isolated Microorganisms Identified In Keratitis Patients. Inventi Impact: Clin. Res., 1-6.

Salviya, L.R., B. Aruna, A. Srinu, Kumar, E.S., Rani, P.R., Lakshmi, D.V., V.R. Prasad, D. 2015. Presumptive identification of clinically important candida sps from oral infection of diabetic patient. Global J. Microbiol. Biotechnol., 3(1): 9-20.

Santos, R.A.C.d., Berretta, A.A., Barud, H.d.S., Ribeiro, S.J.L., GonzálezGarcía, L.N., Zucchi, T.D., RiañoPachóna, D.M. 2014. Draft Genome Sequence of Komagataeibacter rhaeticus Strain AF1, a High Producer of Cellulose, Isolated from Kombucha Tea. Genome Announcements, 2(4).

Sievers, M., Alonso, L., Gianotti, S., Boesch, C., Teuber, M. 1996. 16S23S ribosomal RNA spacer regions of Acetobacter europaeus and A. xylinum, tRNA genes and antitermination sequences. FEMS Microbiol. Lett., 142: 43-48.

Soheir, S., El-Salam, A. 2012. 16S rRNA Gene Sequence Detection of Acetic Acid Bacteria Isolated from Tea Kombucha. New York Sci. J., 5(3): 55-61.

Ukwo, S.P., Ezeama, C.F. 2011. Studies on Proliferation of Acetic Acid Bacteria during Soursop Juice Fermentaion. Int. J. Food Safety, 13: 345-350.

Velićanski, A.S., Cvetković, D.D., Markov, S.L., Šaponjac, V.T.T., Vulić, J.J. 2014. Antioxidant and Antibacterial Activity of the Beverage Obtained by Fermentation of Sweetened 
Lemon Balm (Melissa offi cinalis L.) Tea with Symbiotic Consortium of Bacteria and Yeasts. Food Technol. Biotechnol., 52(4): 420-429.

Yamada, Y., Yukphan, P., Lan Vu, H.T., Muramatsu, Y., Ochaikul, D., Tanasupawat, S., Nakagawa, Y.
2012. Description of Komagataeibacter gen. nov., with proposals of new combinations (Acetobacteraceae). The J. General and Appl. Microbiol., 58(5): 397404.

\section{How to cite this article:}

Tehmeena Ashraf Mukadam, Kapil Punjabi, Sunita D Deshpande, Shashikant Prabhakar Vaidya and Abhay Shadashiv Chowdhary. 2016. Isolation and Characterization of Bacteria and Yeast from Kombucha Tea. Int.J.Curr.Microbiol.App.Sci. 5(6): 32-41.

doi: http://dx.doi.org/10.20546/ijcmas.2016.506.004 